\section{Neue S3-Leitlinie PAVK: Evidenz oft eher schwach}

\author{
Im Jahr 2016 wurde die neue Leitlinie zur PAVK publiziert. Angesichts \\ mangelnder Studiendaten stützen sich viele Empfehlungen lediglich \\ auf Expertenmeinungen und Konsensusvorschläge.
}

„Bei Verdacht auf PAVK gehört neben der Anamnese und klinischen Untersuchung die Messung des Knöchel-ArmIndex in Ruhe zur Basisuntersuchung“, erläuterte Prof. Christiane Tiefenbacher, Wesel. Bei einem Knöchel-Arm-Index (ABI) zwischen 0,9 und 1,3 und tastbaren Pulsen seien bei beschwerdefreien Patienten keine weiteren Untersuchungen mehr erforderlich, da damit die Erkrankung ausgeschlossen sei.

Doch bei Symptomen, abgeschwächten bzw. fehlenden Pulsen, einer Mediasklerose und/oder einem ABI $<0,9$ kommt die farbkodierte Farbdopplersonografie zum Einsatz. Ergibt sich dabei ein aussagefähiger Befund, ist anschließend zur symptomorientierten Therapieplanung im Allgemeinen eine digitale Subtraktionsangiografie (DSA) erforderlich. Bei einem sonografisch nicht eindeutigen Befund empfiehlt sich vor

der DSA zunächst eine MR- oder CTAngiografie.

\section{Therapieempfehlungen}

Als Therapie empfiehlt sich vorrangig der Nikotinstopp (Empfehlungsgrad A, Evidenzlevel 1). Gleiches gilt für Plättchenhemmer (ASS oder Clopidogrel) und Statine zur Prävention kardiovaskulärer Ereignisse. Eine ebenfalls hochrangige Evidenz gibt es für ein strukturiertes Gehtraining unter Aufsicht bei Patienten mit Claudicatio intermittens (CI). „Eine medikamentöse Therapie mit Cilostazol oder Naftidrofuryl sollte nur eingesetzt werden, wenn die Lebensqualität erheblich beeinträchtigt ist, die Gehstrecke unter 200 Meter liegt und ein Gehtraining nicht oder nur eingeschränkt möglich ist“, so Tiefenbacher. Doch dies sei nur eine Konsensusempfehlung. Patienten mit kritischer Ischä-

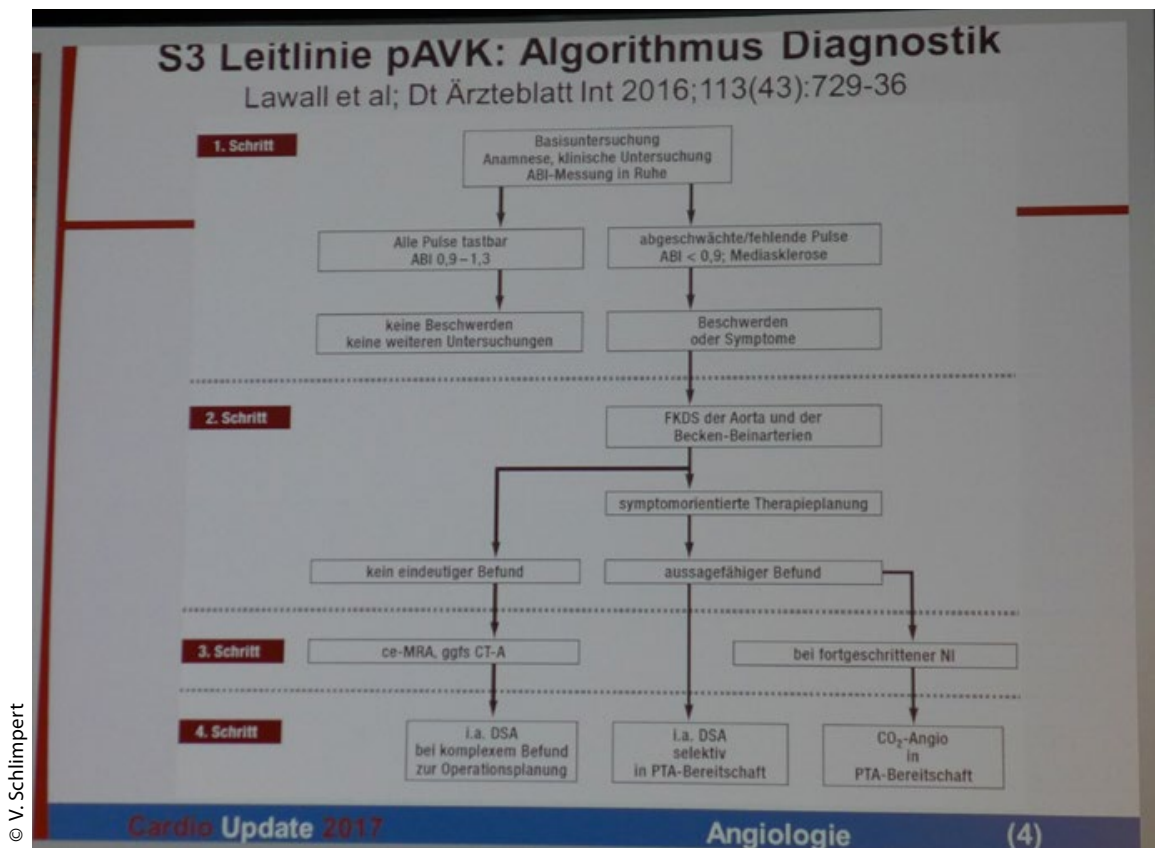

Die Farbdopplersonografie (FKDS) ist absoluter Goldstandard in der Diagnostik der PAVK; ABI: ankle-brachial-index; CT-A: CT-Angiografie; NI: Niereninsuffizienz; DSA: digitale Subtraktionsangiografie; PTA: periphere transluminale Angioplastie. mie oder Infektion sollten antibiotisch behandelt werden (A2-Empfehlung).

Endovaskuläre Verfahren sollten nur Patienten mit CI angeboten werden (Empfehlungsgrad Konsensus mit Evidenzlevel 2), wobei Verschlüsse der aorto-iliacalen Arterien unabhängig vom klinischen Stadium immer zunächst endovaskulär behandelt werden sollten. Gleiches gilt für Verschlüsse der femoropoplitealen Arterien.

„Nur bei langen Verschlüssen, nicht erhöhtem Operationsrisiko, nicht wesentlich eingeschränkter Lebenserwartung und Verfügbarkeit einer Spendervene sollte Bypassverfahren bevorzugt werden", so Tiefenbacher. Auch bei kritischer Extremitätenischämie sollte die endovaskuläre Therapie bevorzugt werden (A2). Nur bei komplexen langstreckigen Verschlüssen der infrapoplitealen Arterien, bei Versagen der endovaskulären Therapie oder Beschwerdepersistenz ist der gefäßchirurgische Eingriff mit dem Empfehlungsgrad Konsensus und dem Evidenzlevel good clinical practice die Therapie der Wahl, soweit eine Bypassvene vorhanden ist.

\section{Welcher Plättchenhemmer?}

Angesichts der Ergebnisse der PLATOund PEGASUS-Studie wurde die Hypothese aufgestellt, dass Ticagrelor bei PAVK effektiver sein könnte als Clopidogrel, ohne die Blutungsrate zu erhöhen. Doch beim direkten Vergleich in der EUCLID-Studie fand sich keine Überlegenheit von Ticagrelor. In beiden Gruppen kam es bei 1,7\% der Patienten zu einer Extremitätenischämie und bei $1,6 \% \mathrm{zu}$ einer schweren Blutung. Auch beim kombinierten primären Endpunkt kardiovaskulärer Tod, Herzinfarkt und ischämischer Schlaganfall gab es keinen Unterschied.

In einer anderen Studie wurde die Kombination des NOAK Edoxaban plus ASS mit ASS plus Clopidogrel verglichen. Die Kombination von Edoxaban plus ASS war sicherer als ASS plus Clopidogrel, d. h. die Blutungsrate war signifikant niedriger. Beim primären Effektivitätsendpunkt Restenose oder Wiederverschluss fand sich nur ein Trend $\mathrm{zu}$ gunsten des NOAK.

sti

Quelle: CardioUpdate, 10.2.2017 in Wiesbaden 